\title{
An loT for Everyone: Fact or Fiction?
}

\author{
Tomás García Ferrari \\ University of Waikato \\ New Zealand \\ tomas.garciaferrari@waikato.ac.nz
}

\author{
Annika Hinze \\ University of Waikato \\ New Zealand \\ annika.hinze@waikato.ac.nz
}

\author{
Judy Bowen \\ University of Waikato \\ New Zealand \\ judy.bowen@waikato.ac.nz
}

\begin{abstract}
The Internet-of-Things (IOT) has the potential to follow a similar progression to Web 2.0, in which end-users came to participate in content creation. This paper analyses the availability of loT components that are suitable for end-users to create their own loT systems with a reasonable degree of ease. Selected IOT platforms are compared using a set of end-user oriented requirements. We critically discuss the current state of the art in this respect and propose a conceptual framework for loT 2.0.
\end{abstract}

Internet of Things (IoT), End-User Development (EUD), Human-Computer Interaction (HCI), interaction design

\section{INTRODUCTION}

The Internet of Things (IoT) is a technological space where physical devices (the "things") generate data and interconnect over the internet. The origins of the term can be trace to a presentation delivered in 1999 by Kevin Ashton (Ashton, 2009). ABI indicates that there will be 40.9 billion internet-enabled devices by 2020 (Drubin, 2014) while Cisco has predicted that there will be 50 billion devices connected by the same time (Evans, 2011).

The personal computer revolution of the '80s brought enthusiasm to many people, not because of technological breakthroughs but because of ease of use. Years later, the World Wide Web was invented. The first web browser was also an editor, but it took several years to arrive at Web 2.0, a paradigmatic change where almost everyone could publish. We understand that a similar scenario could happen with the IoT. In the loT 2.0 space, non-technical users will have the power to create their own data gathering systems and being in control of the generated data.

The paper evaluates some of the loT projects in existence, looking at those that seem to be more accessible to end-users.

\section{INTERNET-OF-THINGS (IOT)}

loT are hardware/software systems that are embedded in everyday objects, communicating via the Internet. These objects with small power supplies, embedded sensors and addresses on the Internet can generate, exchange and consume data with minimum human intervention (Buyya \& Dastjerdi, 2016; Howard, 2015; Rose, Eldridge, \& Chapin, 2015). Fig. 1 shows the data flow of an loT set-up; we will briefly introduce each component type.
Smart physical devices are a mix of electronic hardware components and accompanying software. They comprise sensors, actuators and single-board micro controllers. Sensors can measure the environment (e.g., a thermometer), actuators can interact with the physical world (e.g. a switch). An loT back-end is responsible for data storage and processing. Web and mobile technologies are commonly used for the front-end to allow users to interact with the loT system.

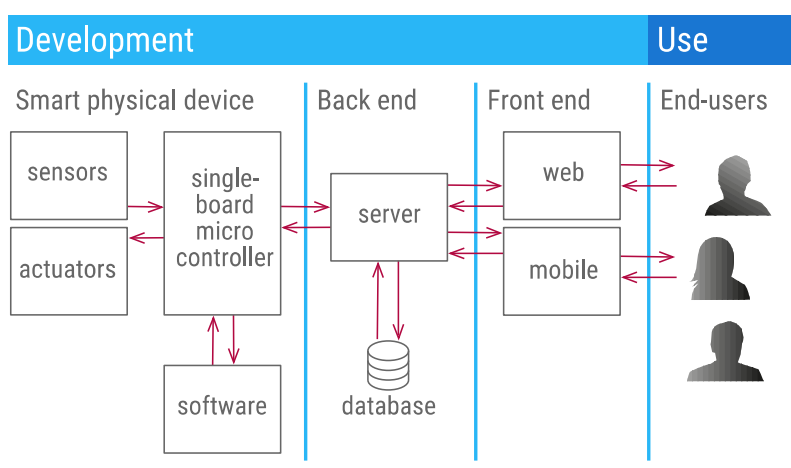

Figure 1: Components of a prototypical loT project.

\section{SCENARIOS AND REQUIREMENT ANALYSIS}

In this section, we present three scenarios and outline our requirements for loT 2.0.

Scenario A: Individual. Jasmine Kirstel is a digital designer working from her home-office. She uses an adjustable desk and wishes to move between standing and sitting positions, but often forgets about changing the table height. She would like to automatically measure the current height of the table during the day and receive alerts on her phone with suggestions to change positions or take a break. In this scenario, data source and use are restricted to one person. 
Scenario B: Family-focused data. The Kendalls like to keep track of their children's height and growth. They see the value in having an easy-touse device to gather these measurements. They want to use a plug and play artefact to measure distance (e.g., from the top of the head to the floor).

In this case, data collection and use are restricted to a relatively small group of people (e.g., between 4 and 12 people).

Scenario C: Citizen Science. The neighbours of Tamatea, in Napier (New Zealand), are unhappy about the noise levels around York Avenue. They want to use a simple system that helps them to acquire, parse and represent the data to expose this problem to the local council. They want to use a set of plug and play artefacts that can be distributed in the neighbourhood and used to measure noise levels.

In this case, a larger group of people (e.g., between $100-500$ people) is potentially interested in data collection and use.

Requirements. From these scenarios we identify the needs for an loT system that adheres to the following conditions:

(i) The things: A collection of artefacts used to measure different dimensions (e.g., distance, temperature, noise).

(ii) Set-up and configuration: The ability to configure the artefacts using the web or a mobile application (through a GUI).

(iii) Interconnectivity: The possibility of connecting to other platforms and services (e.g., SMS).

(iv) Operation: Having artefacts with the possibility of capturing data on request, with the use of a timer, or triggered by an event.

(v) Power: Powered in a way that they can work unattended for long periods of time (e.g., months) not connected to the electricity grid.

(vi) Independence: Work without the constant usage of a device that is not part of the system.

(vii) Visualisation: Offer different ways of visualising the captured data using web and mobile interfaces.

(viii) Connectivity: Work over existing and commonly available networks (e.g., cellular, Wi-Fi).

\section{ANALYSIS OF EXISTING IOT PLATFORMS}

This section analyses existing platforms, from the perspective of what they offer to non-technical users willing to put together an loT project.

Amazon Web Services (AWS) IoT (https://aws.amazon.com/iot) provides the back end, software development kits (SDK) for different devices, a security and encryption layer and the possibility of connecting devices to the cloud, and a way to store and analyse the gathered data.

From the requirements specified under Section 3.4, AWS IoT supports requirement vii, it partially supports requirement iii and similarly to a limited degree requirement $\mathrm{i}$ (it doesn't provide a "thing" but kits that can be used to construct one). It does not cover the rest of the requirements ii, iv, v, vi and viii.

Blynk (http://www.blynk.cc/) is a combination of back- and front-end mobile technologies that allows interaction with smart physical devices over the Internet.

Blynks supports requirements iii and vii, but not i, ii, iv, $v$ and viii.

Cayenne (https://mydevices.com/) presents a drag and drop approach to the process of building loT projects. From the components described in Figure 1 , they offer the back and front end, but they do not provide a smart physical device. The company behind Cayenne, myDevices, offer packaged products called "loT in a Box ${ }^{\mathrm{TM}}$ " where users receive the sensors, a plug \& play gateway and access to the web and mobile apps.

From the requirements specified under Section 3.4, Cayenne supports i, ii, and iii, but it does not offer a good coverage of iv, $v$, vi and vii, which are related to data control and ownership.

Kaa loT (https://www.kaaproject.org/) is an opensource middleware platform. As such, it does not provide any smart physical devices. It could be a good solution if someone wants to implement a cloud system using open-source software, but it does not cover the minimum requirements for endusers with the need to set up an uncomplicated loT project.

Kaa loT only partially supports requirements iii and vii, but it does not support i, ii, iii, iv, vi and viii.

MESH (http://meshprj.com/en/) is a group of small, battery-powered, blocks that combine sensors and electronics and can be used to make smart objects.

MESH supports requirements $i$, ii, iii, iv, v, vii and viii. As the blocks only work associated with a tablet or mobile phone, it does not support vi.

Microsoft loT Central (https://www.microsoft.com/ en-us/internet-of-things/) is a software as a service (SaaS) solution with the goal of providing enterprises with a managed solution for setting loT deployments without the need of starting from scratch. It works with Microsoft cloud products such as Azure loT suite.

MS IoT Central only supports requirement vii, not providing a good solution for i, ii, iii, iv, v and viii.

Particle (https://www.particle.io/) is a company that presents the notion of full-stack loT. They offer two 
different microcontrollers, called Photon (Wi-Fi) and Electron (cellular). The company also provides other hardware solutions, such as an internet button - users can press it to trigger an action and an asset tracker.

Particle supports requirement iii, iv, v, vi, vii and viii, partially ii, and to a certain degree - because these are closed systems - $\mathrm{i}$ with the projects called Internet Button and Asset Tracker.

Smart Citizen (https://smartcitizen.me/) is a platform created to collect data in a participatory manner from an urban environment.

From the requirements specified under Section 3.4, Smart Citizen supports iv, v, vi, vii and viii. The project partially supports $\mathrm{i}$ (by proving a closed and not modular "thing") and it does not support iii.

IBM Watson IoT (https://www.ibm.com/internet-ofthings/) is IBM's platform where people can set up and manage connected devices.

Watson IoT only supports requirement vii, not offering a good solution for i, ii, iii, iv, v, vi and viii (but partially addressing some of them).

Analysis. We have considered nine loT broadspectrum solutions against the set of requirements coming out of our three different scenarios (for an overview of the results see Figure 2).

Summary of findings. Based on this comparison, we can say that the three major industry players (Amazon, Microsoft and IBM) are orienting their efforts to high-end developers. Not many players have focused on providing an excellent coverage of all the areas that we have described as the components of an loT project (Figure 1).

Most platforms offer a certain level of visualisation of the output data (see column 7) but only very few offer devices that could be part of a physical loT system (see column 1). Some of the services, however, can work with loT components provided by the developer/user (see column 3).

Addressing requirements. From our comparison of systems, we see that two of the platforms Particle and Smart Citizen - are very close to cover our set of requirements. Looking into the details, they do not entirely satisfy the need for end-users.

Particle offers products promising that all can be set up in minutes using Particle's mobile app or browser-based setup tools. But just looking at the series of alternatives that they present in their storefront could be a daunting task for nontechnical users. While these products may be good for the enthusiast hobby programmer wanting to prototype an idea, they are not suitable for the endusers in our scenarios.

In the case of the Smart Citizen project, they offer a kit, "an open-source environmental monitoring platform consisting of Arduino-compatible hardware, data visualisation web and mobile app, and a full-featured Rest API." Providing an API is something that attracts developers seeking interoperability between applications, but it is not a feature that end-users understand.

A third system needs mentioning: MESH. It fulfils all requirements besides independence, at it relies on the developer providing a tablet.

Ease of use \& simplicity. During our analysis it became apparent that in addition to the requirements regarding completes of components, an loT 2.0 system would also need to provide a certain ease of use and simplicity in assembling the components.

In the majority of the compared platforms, to put together a project requires a good knowledge in different areas.

From our analysis, we can say that the possibilities offered to end-users have not attained the required level of simplicity. There are some better than others in hiding aspects of the system that are not relevant to these types of users (e.g., MESH is good at hiding the hardware behind colourful blocks).

\begin{tabular}{|c|c|c|c|c|c|c|c|c|}
\hline & 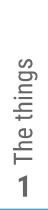 & 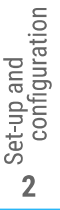 & 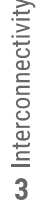 & 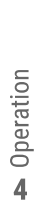 & 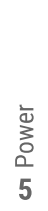 & 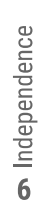 & 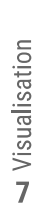 & 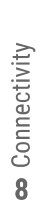 \\
\hline Amazon AWS IoT & $\boldsymbol{x}$ & $\boldsymbol{x}$ & $?$ & $\boldsymbol{x}$ & $\boldsymbol{x}$ & $\boldsymbol{x}$ & 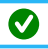 & $\boldsymbol{x}$ \\
\hline Blynk & $\boldsymbol{x}$ & $\boldsymbol{x}$ & $\nabla$ & $\boldsymbol{x}$ & $\boldsymbol{x}$ & $\boldsymbol{x}$ & 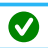 & $\boldsymbol{x}$ \\
\hline Cayenne & $\nabla$ & $\nabla$ & $\nabla$ & $\boldsymbol{x}$ & $\nabla$ & $\nabla$ & $\nabla$ & $\nabla$ \\
\hline Kaa loT & $\boldsymbol{x}$ & $\boldsymbol{x}$ & $?$ & $\boldsymbol{x}$ & $\boldsymbol{x}$ & $\boldsymbol{x}$ & $?$ & $\boldsymbol{x}$ \\
\hline Mesh & 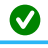 & $\nabla$ & 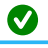 & $\nu$ & $\boldsymbol{\nu}$ & $\boldsymbol{x}$ & $\nabla$ & $\nabla$ \\
\hline Microsoft loT Central & $\boldsymbol{x}$ & $\boldsymbol{x}$ & $\boldsymbol{x}$ & $\boldsymbol{x}$ & $\boldsymbol{x}$ & $\boldsymbol{x}$ & $\nabla$ & $\boldsymbol{x}$ \\
\hline Particle & $\theta$ & $\theta$ & 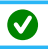 & $\boldsymbol{\nu}$ & 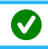 & $\varnothing$ & 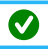 & $\nabla$ \\
\hline Smart Citizen & 8 & 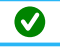 & $?$ & $\boldsymbol{\nu}$ & $\boldsymbol{\nu}$ & $\nabla$ & $\nabla$ & $\nu$ \\
\hline IBM Watson loT & $\boldsymbol{x}$ & $\boldsymbol{x}$ & $\boldsymbol{x}$ & $\boldsymbol{x}$ & $\boldsymbol{x}$ & $\boldsymbol{x}$ & $\nabla$ & $\boldsymbol{x}$ \\
\hline
\end{tabular}

Figure 2: Comparing loT platforms. The green icon $\downarrow$ indicates that the evaluated platform covers the evaluated condition, the amber icon? shows that it is not explicit or documented, while the red icon $\boldsymbol{\otimes}$ means that the platform does not cover it. The green and red combined icon indicates that the platform includes it with limitations.

\section{DISCUSSION}

Considering that we are at the early days of the Internet of Things (Bröring, Datta, \& Bonnet, 2016), IoT technologies are discussed with considerable depth and breadth in professional and academic venues. Nevertheless, end-users factors are still 
not thoroughly addressed with respect to building loT projects.

Summary of findings. The findings in our comparative analysis of loT platforms indicate that while many of them are promising simplicity and ease of use, they are clearly targeting developers.

In many cases, loT technologies are built to be used in specific, condoned ways (Jenkins, 2015). Our analysis shows that while there are many available technical solutions, they do not include the ease of use which would help drive the equivalent of the personal computer revolution or Web 2.0 for loT.

An IoT 2.0 Conceptual Framework. It is our conclusion that a successful loT 2.0 approach needs a clearer pathway for an end-user to develop their own loT system. This includes ease of use of existing components as well as simplicity in assembling.

We will briefly describe here our concept for how such help may be provided.

1. First, end-users need tools that help them to map their loT 2.0 ideas. At this stage, a user may have a general goal in mind, but not yet have a clear concept of, for example, which data needs to be captured.

2. Secondly, users need to identify conceptually, which types of components they wish to use.

3. Thirdly, the technical parts (sensors, actuators, controller, etc.) need to be presented in a truly plug-and-play form, to be combined to implement their concept from step 2.

We are in the process of developing two sets of cards that allow end users to be guided through steps 1 and 2. While there exist already cards for ideating and developing loT projects ("Learn. Play. Collect.," n.d.; "The loT Design Deck - A Co-Design method for the connected products," n.d.), they mostly target expert developers and programmers. Finally, there needs to be a set of truly plug-and-play sensors, actuators and controllers. This means that there should not be any parts that have open electronic connections that need soldering. Finally, there needs to be a guide on how to assemble the elements.

\section{CONCLUSIONS}

In this paper, we compared nine loT projects for their suitability to support the IoT 2.0 concept in which end-users (not professional developers) can assemble loT systems. None of the analysed projects seem to be targeting to empower endusers, as they do not provide the required components nor the necessary simplicity and ease of use.
At the early stage of any technological development, systems evolve according to what the technology is capable of offering, and user considerations typically came at a later stage. We believe that we have surpassed this first phase in the case of loT, but to date, no system offers all aspects of technical support while providing simplicity and ease of composition as needed for the second phase leading into IOT 2.0. Under these conditions, the idea of an loT for everyone seems to be still in the fictional area.

Finally, we identify a question that needs to be addressed: will the architecture of the Internet of Things be a system of freedom or a method of control? In the context of technology, most of the developments can be used for good but also for making things worse. There is a good chance that the loT presents a bottom-up approach that will create new emergent phenomena (Johnson, 2004). But the indications of adopting a top-down architecture are also there. The current state of affairs is not necessarily guaranteeing good results.

For future work, we are planning to complete and refine our initial set of cards that will support steps 1 and 2. As new loT development systems appear, we will continue to evaluate them according to the criteria we developed here. We are also currently engaged in field studies, testing conceptual cards with non-technical users to conceptually identify the components they wish to use. More research in this area will enable us to understand if, confronted with excellent opportunities, end users may embrace the idea of creating their own independent loT systems.

\section{REFERENCES}

Ashton, K. (2009). That 'internet of things' thing. RFiD Journal, 22(7), 97-114.

Bröring, A., Datta, S. K., \& Bonnet, C. (2016). A Categorization of Discovery Technologies for the Internet of Things (pp. 131-139). ACM Press. https://doi.org/10.1145/2991561.2991570

Buyya, R., \& Dastjerdi, A. V. (2016). Internet of Things: principles and paradigms. Amsterdam, Boston, Heidelberg: Morgan Kaufmann.

Drubin, C. (2014). The internet of things will drive wireless connected devices to. 40.9 billion in 2020. Microwave Journal, 57(10), 51.

Evans, D. (2011). The Internet of Things. Cisco Internet Business Solutions Group (IBSG).

Howard, P. N. (2015). Pax technica: how the internet of things may set us free or lock us up. New Haven, London: Yale University Press.

Jenkins, T. (2015). Designing the "Things" of the loT (pp. 449-452). ACM Press. https://doi.org/10.1145/2677199.2691608 
Johnson, S. (2004). Emergence: the connected lives of ants, brains, cities and software. New York, NY: Scribner.

Learn. Play. Collect. (n.d.). Retrieved May 25, 2018, from https://know-cards.myshopify.com/

Rose, K., Eldridge, S., \& Chapin, L. (2015). The internet of things: An overview. The Internet Society (ISOC), 1-50.

The loT Design Deck - A Co-Design method for the connected products. (n.d.). Retrieved May 25, 2018, from http://www.iotdesigndeck.com/ 